\title{
XXXVII. Electromagnets.-III. Iron and steel. New theory of magnetism
}

\section{R.H.M. Bosanquet}

To cite this article: R.H.M. Bosanquet (1885) XXXVII. Electromagnets.-III. Iron and steel. New theory of magnetism , Philosophical Magazine Series 5, 19:120, 333-340, DOI: $10.1080 / 14786448508627684$

To link to this article: http://dx.doi.org/10.1080/14786448508627684

册 Published online: 29 Apr 2009.

Submit your article to this journal $[\pi$

Џ Article views: 2

Q View related articles $\asymp$ 
XXXVII. Electromagnets.-III. Iron and Steel. New Theory of Magnetism. By R. H. M. Bosanquet, St. John's College, Oxford.

To the Editors of the Philosophical Magazine and Journal.

GeNTLemen,

TN the February number of the Philosophical Magazine 1 (p. 73) I gave a number of experiments on the Permeability $(\mu)$ of Iron and Steel, with empirical formulæ founded on Fourier's Series. I also gave the elements of a new theory leading to equations of the form

$$
\begin{aligned}
\mu & =\mathbf{A}\left(\mathfrak{B}_{\infty}-\mathfrak{B}\right) \cos \delta, \\
\delta & =f \theta, \\
\mathfrak{B} & =\frac{k}{\rho} \frac{60^{\circ}-\omega \theta}{\sin \theta},
\end{aligned}
$$

and gave the comparison of this theory with experiment in the cases of Crown (soft bar) Iron ring $\mathbf{E}$, and Steel ring $\mathbf{J}$ (first soft state). I propose now to communicate the comparisons of all the remaining experiments of the former paper, and of three additional sets on J.

For clearness I will recapitulate shortly the bearing of the formulæ.

Each molecule has one, and only one, axis of transmission (like* a bead with a hole in it). The axis is capable of transmitting $\mathbb{B}_{\infty}$ lines of force and no more, and the molecular permeability is proportional to $\mathbf{B}_{\infty}-\mathbf{a b}$, or to the defect of saturation. (If we pack the hole in the bead with thin wires, the aperture remaining is represented by the number of wires that remain to be got in.)

$A$ is the molecular permeability per unit defect of saturation.

$\delta$ is an auxiliary angle representing the obliquity of a zigzag which would augment the resistance to the amount actually observed.

$\theta$ is the average inclination of the axes of the molecules to the direction of magnetization; initially it is always $60^{\circ}$. $\omega$ is the unit angle. $f$ is the factor connecting $\theta$ and $\delta$.

$\frac{k}{\rho}$ represents the force of molecular torsion which is in equilibrium with the tendency of the lines of force further to deflect the average molecule.

* These statements are not to be taken as actual hypotheses, but as geometrical analogies of the distribution.

Phil. Mag. S. 5. Vol. 19. No. 120. May 1885. 2 A 
The inferences as to these constants to be drawn from the numerous experiments now discussed are not so clear as they appeared to be from the two cases treated in the last paper.

The first of the following Tables contains the constants from all the experiments that have been reduced, finishing with those obtained from Rowland's Table I.

The remaining Tables contain the detailed comparison of the theory with experiment in all the cases, except ring $E$, and $\mathbf{J}$ first soft state, which two were given in the last paper.

The last column but one of the first Table gives the product of $A$, the reciprocal of $\frac{\kappa}{\rho}$, and a constant. This quantity may be compared with the maximum observed value of $\mu$ in the last column.

A has been spoken of as the molecular permeability for a certain unit. Regarding magnetism as a motion or displacement, whether dynanic or static, we may thus speak of $A$ as a coefficient of freedom within the molecule.

$\frac{\kappa}{\rho}$ being the coefficient of the forces which tend to prevent the rotation of the molecule as a whole, we may speak of its reciprocal as a coefficient of freedom without the molecule.

The last column but one may be therefore regarded as the product of coefficients of intra-molecular and extra-molecular freedom, and a constant.

This product $(c \times$ intra- $\times$ extra-molecular freedom $)$ is a characteristic of a given approximate state of a given piece of metal. It runs generally with the maximum of $\mu$; but is liable to be depressed with respect to $\mu$ where $f$ has high values, as in the first, second, and fourth entries. As between the hard steel and the iron, the product of the coefficients of freedom is proportional to the maximum permeability.

The further general conclusions, so far as these data go, are as follows:-

In soft steel the molecular forces are chiefly extra-molecular, the freedom intra-molecular.

In hard steel the molecular forces are chiefly intra-molecular, the freedom extra-molecular.

In soft iron the average intra-molecular freedom is nuch greater than in hard steel, the extra-molecular freedom about the same.

In soft steel the extra-molecular freedom is much diminished, the intra-molecular freedom is moderate or high.

The distribution of the freedom between intra- and extramolecular conditions depends minutely on the condition of the piece of iron or steel. 
The variations thus arising are comparatively moderate in iron, considerable in hard steel, and enormous in soft steel.

In one or two cases the equations do not represent the values quite so well; in $\mathrm{G}$ two sets of constants are given.

The dimensions of the rings and details of the experiments are given in my paper of February last.

Steel ring $J$ was examined first soft, then hard, then soft again, then hard again. Its saturation-point is taken to be 20,000 throughout. It was wound with about 4000 turns in the hard states.

P.S.-From the above conclusions we can deduce the outline of a chemical theory of the hardening and tempering of steel.

In hard steel the additional constraint, as compared with iron, is wholly intra-molecular, $i$. e. due to chemical combination with a steeling element. This combination must exist naturally at a red heat, and be stereotyped by sudden cooling.

In soft steel the intra-molecular constraint is much diminished, and the extra-molecular constraint increased. Hence in slow cooling dissociation of the steeling element takes place. This requires time. At any point in the slow cooling the condition of partial dissociation can be stereotyped by completing the cooling suddenly. This constitutes tempering.

Scleme of the Constants of the Rings.

J. Steel.--E, F, G, H, K. Soft Iron (Crown). I. Lowmoor. Rowland's I., "Burden's Best."

\begin{tabular}{|c|c|c|c|c|c|}
\hline Ring. & A. & $\log \frac{\kappa}{\rho}$. & $\log f$. & $\begin{array}{c}\frac{A \rho}{\kappa} \times C \\
\log \mathrm{C}=61000 .\end{array}$ & $\begin{array}{l}\text { Maximum } \\
\text { observed } \\
\text { ralue of } \mu .\end{array}$ \\
\hline J. First soft state... & 11. & $4 \cdot 78936$ & $\cdot 17595$ & 225 & 461 \\
\hline "Second soft state & $\cdot 129$ & 282178 & $\cdot 16552$ & 245 & 423 \\
\hline "First hard state. & $\cdot 01735$ & $2 \cdot 16327$ & .13925 & 150 & 157 \\
\hline "Second hard state & $\cdot 0236$ & $2 \cdot 46312$ & $\cdot 15340$ & 102 & 145 \\
\hline $\mathrm{E} \quad \ldots \ldots \ldots \ldots \ldots \ldots$ & $\cdot 3856$ & 232208 & $\cdot 16153$ & 2312 & $223 \pm$ \\
\hline $\bar{F} \ldots \ldots$. & $\cdot 189$ & 201524 & $\cdot 16151$ & 2297 & 1895 \\
\hline G & 35 & $2 \cdot 23407$ & $\cdot 15778$ & $2570\}$ & 2501 \\
\hline H & .31 & $2 \cdot 11959$ & .15772 & $2963 \mathrm{~J}$ & 1707 \\
\hline $\begin{array}{l}\mathbf{n} \\
\mathbf{K}\end{array}$ & .39 & 2.30682 & .15973 & 2422 & 2070 \\
\hline$\vec{I} \quad \ldots \ldots \ldots \ldots \ldots \ldots \ldots$ & .217 & $2 \cdot 06175$ & $\cdot 16046$ & 2370 & 2173 \\
\hline Rowland's Tabble I. & 343 & $2 \cdot 14941$ & $\cdot 16079$ & 2666 & 2472 \\
\hline
\end{tabular}


J. First Hard State.

$\mathrm{A}=\cdot 01735, \quad \log \frac{\kappa}{\rho}=2 \cdot 16327, \quad \log f=\cdot 13925$.

$\mathfrak{B}_{\infty}=20,000$.

\begin{tabular}{|c|c|c|c|c|c|}
\hline \multirow{2}{*}{33.} & \multirow{2}{*}{$\theta$. } & \multirow{2}{*}{$\delta$} & \multicolumn{2}{|c|}{$\mu$} & \multirow{2}{*}{ Diffs. } \\
\hline & & & Oale. & Obs. & \\
\hline $\begin{array}{r}13 \\
59 \\
121 \\
170 \\
268 \\
405 \\
551 \\
645 \\
2189 \\
4492 \\
6496 \\
8959 \\
13870 \\
16292 \\
19533\end{array}$ & $\begin{array}{rr}59 & 56 \\
59 & 39 \\
59 & 17 \\
59 & 0 \\
58 & 26 \\
57 & 40 \\
56 & 50 \\
56 & 20 \\
48 & 40 \\
40 & 8 \\
34 & 39 \\
29 & 36 \\
22 & 55 \\
20 & 36 \\
18 & 0\end{array}$ & $\begin{array}{ll}82 & 35 \\
82 & 11 \\
81 & 41 \\
81 & 18 \\
80 & 31 \\
79 & 38 \\
78 & 19 \\
77 & 38 \\
67 & 4 \\
55 & 18 \\
47 & 45 \\
40 & 47 \\
31 & 35 \\
28 & 23 \\
24 & 48\end{array}$ & $\begin{array}{r}45 \\
47 \\
50 \\
52 \\
56 \\
62 \\
68 \\
72 \\
120 \\
153 \\
157 \\
145 \\
91 \\
57 \\
7\end{array}$ & $\begin{array}{r}49 \\
47 \\
49 \\
50 \\
57 \\
62 \\
64 \\
67 \\
115 \\
150 \\
157 \\
134 \\
70 \\
56 \\
53\end{array}$ & $\begin{array}{r}-5 \\
0 \\
+1 \\
+2 \\
+1 \\
0 \\
+4 \\
+5 \\
+5 \\
+3 \\
+11 \\
+21 \\
+1 \\
+45\end{array}$ \\
\hline
\end{tabular}

J. Second Soft State.

$\mathrm{A}=\cdot 129, \quad \log \frac{\kappa}{\rho}=2 \cdot 82178, \quad \log f=\cdot 16552$.

$\mathbf{B}_{\infty}=20,000$.

\begin{tabular}{|c|c|c|c|c|c|}
\hline \multirow{2}{*}{ B. } & \multirow{2}{*}{$\theta}$. & \multirow{2}{*}{$\delta}$. & \multicolumn{2}{|c|}{$\mu}$. & \multirow{2}{*}{ Diffs. } \\
\hline & & & Calc. & Obs. & \\
\hline 44 & 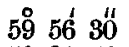 & $87^{\circ} 45$ & 101 & 92 & +9 \\
\hline 348 & $59 \quad 3252$ & 8710 & 125 & 125 & \\
\hline 1110 & 5835 & 8546 & 180 & 188 & -8 \\
\hline 1464 & 587 & 855 & 205 & 201 & +4 \\
\hline 1640 & 5755 & $84 \quad 47$ & 215 & 211 & +4 \\
\hline 2377 & 570 & 8327 & 259 & 261 & -2 \\
\hline 3701 & 5524 & 816 & 325 & 323 & +2 \\
\hline 6734 & 520 & 767 & 411 & 422 & -11 \\
\hline 9957 & 4843 & $71 \quad 19$ & 415 & 423 & -8 \\
\hline 10029 & 4840 & 7115 & 413 & 353 & +60 \\
\hline 13252 & 4542 & 6654 & 341 & 334 & +7 \\
\hline 14470 & 4440 & 6523 & 295 & 227 & +68 \\
\hline 16118 & 4320 & 6326 & 224 & 173 & +51 \\
\hline 19198 & 410 & 601 & 52 & 61 & -9 \\
\hline
\end{tabular}


New Theory of Magnetism.

J. Second Hard State.

$A=\cdot 0236 . \quad \log \frac{\kappa}{\rho}=2 \cdot 46312, \quad \log f=\cdot 15340$.

$\mathbf{B B}_{\infty}=20,000$.

\begin{tabular}{|c|c|c|c|c|c|}
\hline \multirow{2}{*}{ BB. } & \multirow{2}{*}{$\theta$. } & \multirow{2}{*}{$\delta}$. & \multicolumn{2}{|c|}{$\mu}$. & \multirow{2}{*}{ Diffs. } \\
\hline & & & Calo. & Obs. & \\
\hline 43 & 59852 20 & 8514 & 39 & 39 & \\
\hline 204 & $\begin{array}{lll}59 & 33 & 40\end{array}$ & $84 \mathbf{3 3}$ & 44 & 39 & +5 \\
\hline $5: 38$ & 5826 & 8311 & 54 & 46 & +8 \\
\hline 821 & 5736 & 820 & 63 & 58 & +5 \\
\hline 923 & 5720 & 8137 & 66 & 59 & +7 \\
\hline 1308 & 5615 & 805 & 76 & 72 & +4 \\
\hline 1489 & 5546 & $\begin{array}{ll}79 & 24\end{array}$ & 80 & 77 & +3 \\
\hline 1572 & 5532 & $\begin{array}{ll}79 & 4\end{array}$ & 82 & 78 & +4 \\
\hline 1767 & $55 \quad 1$ & 7818 & 87 & 81 & +6 \\
\hline 2186 & 5355 & 7646 & 96 & 106 & -10 \\
\hline 2477 & 5310 & 7541 & 102 & 113 & -11 \\
\hline 3655 & 5020 & 7139 & 121 & 130 & -9 \\
\hline 4305 & 4850 & 6931 & 130 & 133 & -3 \\
\hline 4468 & 4830 & 693 & 131 & 136 & -5 \\
\hline 4822 & 4743 & 6756 & 134 & 142 & -8 \\
\hline 5442 & 4626 & 666 & 139 & 142 & -3 \\
\hline 6255 & 4450 & 6350 & 143 & 143 & o \\
\hline 7351 & $49 \quad 48$ & 6056 & 145 & 145 & 0 \\
\hline 8793 & $40 \quad 24$ & 5731 & 142 & 142 & 0 \\
\hline 11128 & 3658 & 5238 & 124 & 123 & +1 \\
\hline 12631 & 352 & 4952 & 112 & 97 & +15 \\
\hline 14093 & 3320 & 4727 & 94 & 86 & +8 \\
\hline 15515 & 3150 & $45 \quad 19$ & 74 & 66 & +8 \\
\hline 18276 & 2916 & 4140 & 30 & 48 & -18 \\
\hline
\end{tabular}

F.

$\mathrm{A}=\cdot 189, \quad \log \frac{\kappa}{\rho}=2 \cdot 01524, \quad \log f=\cdot 16151$.

$\mathbf{B}_{\infty}=20,000$.

\begin{tabular}{|c|c|c|c|c|c|}
\hline \multirow{2}{*}{ B. } & \multirow{2}{*}{$\theta$. } & \multirow{2}{*}{$\delta$} & \multicolumn{2}{|c|}{$\mu}$. & \multirow{2}{*}{ Diffs. } \\
\hline & & & Oalc. & Ohs. & \\
\hline 15 & 59852 30́ & $86^{\circ} 51^{\prime}$ & 208 & 208 & \\
\hline 143 & 5849 & 8519 & 306 & 360 & -54 \\
\hline 513 & 5540 & 8045 & 592 & 587 & +5 \\
\hline 2784 & 420 & 6055 & 1582 & 1592 & -10 \\
\hline 3616 & $38 \quad 22$ & 5539 & 1747 & 1784 & -37 \\
\hline 4283 & 3548 & 5156 & 1832 & 1869 & -37 \\
\hline 5747 & $31 \quad 15$ & 4520 & 1894 & 1895 & -1 \\
\hline 6438 & $29 \quad 27$ & 4243 & 1883 & 1825 & +58 \\
\hline 7655 & 2645 & 3848 & 1818 & 1704 & +104 \\
\hline 10767 & 2140 & 3126 & 1489 & 1581 & -92 \\
\hline 12537 & 1934 & 2823 & 1241 & 1252 & -11 \\
\hline 13900 & $1810^{\circ}$ & 2621 & 1033 & 1000 & +33 \\
\hline 15035 & $17 \quad 11$ & 2455 & 853 & 692 & +161 \\
\hline 18834 & 1431 & 213 & 206 & 150 & +56 \\
\hline
\end{tabular}


G.

$$
\begin{array}{cc}
\Delta=\cdot 35, & A=\cdot 31, \\
\log \frac{\boldsymbol{\alpha}}{\rho}=2 \cdot 23407, \log f=\cdot 15778 . & \log _{\rho}^{\boldsymbol{\alpha}}=2 \cdot 11959, \log f=\cdot 15572 . \\
\mathfrak{B B}_{\infty}=18,000 . & \mathfrak{B}_{\infty}=18,000 .
\end{array}
$$

\begin{tabular}{|c|c|c|c|c|c|c|c|c|}
\hline \multirow{2}{*}{ 球. } & \multirow{2}{*}{$\theta}$. & \multirow{2}{*}{$\delta}$. & \multicolumn{2}{|c|}{$\mu$. } & \multirow{2}{*}{ Diffs. } & \multicolumn{2}{|c|}{$\mu}$. & \multirow{2}{*}{ Diffs. } \\
\hline & & & Calc. & Obs. & & Calc. & Obs. & \\
\hline 51 & 594440 & 8555 & 447 & 461 & -14 & 446 & 461 & -15 \\
\hline 448 & 5748 & 837 & 736 & 765 & -29 & 778 & 765 & +13 \\
\hline 1972 & 513 & 7325 & 1601 & 1615 & -14 & 1738 & 1615 & +123 \\
\hline 6364 & 3726 & 5350 & 2403 & 2501 & -98 & 2423 & 2501 & -78 \\
\hline 9003 & 336 & 4736 & 2123 & 2241 & -118 & 2136 & 2241 & -105 \\
\hline 11090 & 2850 & 4128 & 1812 & 1763 & +49 & 1745 & 1763 & -18 \\
\hline 11970 & 2738 & 3944 & 1623 & 1555 & +68 & 1553 & 1555 & -2 \\
\hline 13710 & 2530 & 3640 & 1204 & 1192 & +12 & 1140 & 1192 & -52 \\
\hline 14426 & $24 \quad 46$ & 3537 & 1017 & 832 & +185 & 974 & 832 & +142 \\
\hline & 2341 & $34 \quad 3$ & 724 & 664 & +60 & 680 & 664 & +16 \\
\hline 16042 & 2311 & 3154 & 562 & 395 & +167 & 537 & 395 & +142 \\
\hline 17536 & 2153 & 3128 & 138 & 145 & -7 & 129 & 145 & +16 \\
\hline
\end{tabular}

\begin{tabular}{|c|c|c|c|c|c|}
\hline \multirow{2}{*}{ BB. } & \multirow{2}{*}{$\theta$. } & \multirow{2}{*}{$\delta}$. & \multicolumn{2}{|c|}{$\mu$} & \multirow{2}{*}{ Diffs. } \\
\hline & & & Cale. & Obs. & \\
\hline 34 & 5950 & 85 & 380 & 395 & -15 \\
\hline 287 & 5836 & 8324 & 509 & 418 & +91 \\
\hline 1079 & $5 \pm 56$ & 7811 & 866 & 817 & +49 \\
\hline 6025 & $\begin{array}{ll}38 \quad 29\end{array}$ & 5456 & 1727 & 1797 & -70 \\
\hline 7705 & 3446 & 4919 & 1678 & 1717 & -39 \\
\hline 8436 & 3322 & $47 \quad 29$ & 1616 & 1710 & -94 \\
\hline 9740 & 316 & 4416 & 1479 & 1354 & +125 \\
\hline 11261 & 2850 & $41 \quad 2$ & 1271 & 1134 & +137 \\
\hline 13355 & 2610 & $37 \quad 14$ & 903 & 885 & +18 \\
\hline 14501 & 2455 & 3528 & 712 & 630 & +82 \\
\hline 14718 & 2440 & $\begin{array}{ll}35 & 1\end{array}$ & 672 & 502 & +170 \\
\hline 15409 & 240 & $\begin{array}{ll}34 & 9\end{array}$ & 536 & 254 & +282 \\
\hline 17642 & 220 & $\begin{array}{lll}31 & 18\end{array}$ & 76 & 97 & -21 \\
\hline
\end{tabular}

\section{H.}

$$
\mathrm{A}=\cdot 25, \quad \log \frac{\kappa}{\rho}=2 \cdot 24075, \quad \log f=\cdot 15325 .
$$

$\mathfrak{B B}_{\infty}=18,000$. 
$\mathrm{K}$.

$\mathrm{A}=\cdot 39, \quad \log _{\rho} \frac{\kappa}{\rho}=2 \cdot 30682, \quad \log f=\cdot 15973$.

$\mathbf{B B}_{\infty}=15,500$.

\begin{tabular}{|c|c|c|c|c|c|}
\hline \multirow{2}{*}{ 解. } & \multirow{2}{*}{$\theta$} & \multirow{2}{*}{$\delta$} & \multicolumn{2}{|c|}{$\mu$} & \multirow{2}{*}{ Diffs. } \\
\hline & & & Calc. & Obs. & \\
\hline 67 & $5 \stackrel{\circ}{9} 42$ & $86^{\circ} 16$ & 391. & 423 & -31 \\
\hline 293 & 5846 & 8453 & 520 & $4: 3$ & +101 \\
\hline 2175 & 5136 & 7432 & 1380 & 1:88 & +98 \\
\hline 2337 & 512 & $7 \overline{3} 4 \overline{3}$ & 1439 & 1176 & +263 \\
\hline 3949 & 460 & 6627 & 1800 & 1766 & +34 \\
\hline 4710 & 4353 & 6323 & 1982 & 1885 & +97 \\
\hline 5720 & 412130 & 5945 & 1922 & 2070 & $-1+8$ \\
\hline 8677 & 3517 & 5058 & 1676 & 1914 & -238 \\
\hline 8890 & 3454 & 5025 & 1643 & 1775 & -132 \\
\hline 9598 & 3343 & $48 \quad 43$ & 1519 & 1557 & -38 \\
\hline 10413 & 3227 & 4652 & 1356 & 1531 & -175 \\
\hline 11511 & 305230 & 4436 & 1107 & 1104 & +3 \\
\hline 12148 & 301 & 4322 & 950 & 799 & +151 \\
\hline 13246 & 2840 & 4125 & 659 & 666 & -7 \\
\hline 13104 & 2850 & 4139 & 698 & 505 & +193 \\
\hline 13671 & 2310 & 4041 & 541 & 358 & +183 \\
\hline 15053 & 2640 & 3831 & 136 & 135 & $+\quad 1$ \\
\hline
\end{tabular}

I.

$\mathrm{A}=\cdot 217, \quad \log \frac{\kappa}{\rho}=2 \cdot 06175, \quad \log f=\cdot 16046$.

$\mathbf{b}_{\infty}=20,000$.

\begin{tabular}{|c|c|c|c|c|c|}
\hline \multirow{2}{*}{$\mathfrak{B}$} & \multirow{2}{*}{$\theta}$. & \multirow{2}{*}{$\delta}$. & \multicolumn{2}{|c|}{$\mu}$. & \multirow{2}{*}{ Diffs. } \\
\hline & & & Calc. & Obs. & \\
\hline 33 & $59945^{\prime}$ & 8627 & 268 & 271 & -3 \\
\hline 429 & $56 \quad 5230$ & 8218 & 569 & 617 & -48 \\
\hline 1322 & 515 & 7355 & 1123 & 1024 & +99 \\
\hline 1624 & 4920 & 7123 & 1273 & 1427 & -154 \\
\hline 2395 & 4515 & $65 \quad 29$ & 1585 & 1558 & +27 \\
\hline 4541 & 3633 & 5253 & 2024 & 2072 & -48 \\
\hline 6324 & 3125 & 4527 & 2083 & 2080 & +2 \\
\hline 6712 & $30 \quad 29$ & 446 & 2071 & 1993 & +78 \\
\hline 6700 & 3030 & 448 & 2071 & 2033 & +38 \\
\hline 7091 & 2937 & 4251 & 2054 & 2173 & -119 \\
\hline 8194 & 272130 & 3935 & 1974 & 1935 & +39 \\
\hline 9691 & 2447 & $35 \quad 52$ & 1813 & 1842 & -29 \\
\hline 10400 & $23 \quad 43 \quad 30$ & 3420 & 1720 & 1483 & +237 \\
\hline 13159 & 2020 & 2925 & 1293 & 1188 & +105 \\
\hline 15050 & 1832 & $26 \quad 49$ & 958 & 818 & +140 \\
\hline 16309 & 1730 & 2519 & 724 & 592 & +132 \\
\hline 16939 & 170 & 2436 & 604 & 444 & +160 \\
\hline 19303 & 1434 & 215 & 141 & 155 & -14 \\
\hline
\end{tabular}


Rowland's Table I.

$\mathrm{A}=\cdot 343, \quad \log \frac{\kappa}{\rho}=2 \cdot 14941, \quad \log f=\cdot 16079 . \quad \mathbf{B}_{\infty}=17,500$.

\begin{tabular}{|c|c|c|c|c|c|}
\hline \multirow{2}{*}{ HB. } & \multirow{2}{*}{$\theta$. } & \multirow{2}{*}{$\delta}$. & \multicolumn{2}{|c|}{$\mu}$. & \multirow{2}{*}{ Diffs. } \\
\hline & & & Calc. & Obs. & \\
\hline 71.5 & $5 \stackrel{9}{9} 3 \dot{4}$ & $86^{\circ} 15$ & 391 & 391 & 0 \\
\hline 600.5 & $\begin{array}{ll}56 & 27\end{array}$ & 8144 & 833 & $\begin{array}{l}091 \\
869\end{array}$ & -36 \\
\hline $966 \cdot 7$ & 5427 & 7851 & 1097 & 1129 & -32 \\
\hline 2460 & $47 \quad 12$ & 6821 & 1903 & 1936 & -33 \\
\hline 2923 & 4517 & $\begin{array}{lll}65 & 34\end{array}$ & 2068 & 2078 & -10 \\
\hline 3082 & 4438 & 6438 & 2119 & 2124 & -5 \\
\hline 4959 & $38 \quad 15$ & 5523 & 2444 & 2433 & +11 \\
\hline 5482 & 3645 & 5313 & 2468 & 2470 & -2 \\
\hline 5782 & 3556 & $52 \quad 2$ & 2473 & 2472 & $+\overline{1}$ \\
\hline 6651 & 3347 & $48 \quad 55$ & 2445 & 2448 & $-\overline{3}$ \\
\hline 7473 & 3158 & $\begin{array}{ll}46 \quad 17\end{array}$ & 2371 & 2367 & +4 \\
\hline 8943 & 298 & 4211 & 2175 & 2208 & -33 \\
\hline 10080 & 2716 & 3929 & 1964 & 1899 & +65 \\
\hline 12270 & 2416 & 358 & 1467 & 1448 & $\begin{array}{l}19 \\
+19\end{array}$ \\
\hline 12970 & $23 \quad 24$ & 3353 & 1290 & 1269 & +21 \\
\hline 13630 & 2242 & 3252 & 1115 & 1137 & -22 \\
\hline 14540 & 2145 & 3130 & 866 & 824 & +42 \\
\hline 15770 & 2037 & 2951 & 515 & 462 & +53 \\
\hline 16270 & 2012 & $\begin{array}{lll}29 & 15\end{array}$ & 368 & 354 & +14 \\
\hline 16600 & 1949 & $28 \quad 42$ & 271 & 258 & +13 \\
\hline
\end{tabular}

XXXVIII. On the Seat of the Electromotive Forces in the Voltaic Cell. By Professor OLIver J. Lodge, D.Sc.

[Concluded from p. 280.]

Table of Contents.

Chapter IV. Discursive*.

19. Discussion and hypothetical explanation of true (or thermoelectric) contact-force, and reason why for insulators it is large, and for metals small. ........................ 340

20. Thermoelectric contact-forces between metals and electrolytes, and theory of the common or simple voltaic cell .......... 343

21. Experimental examination of three simple cells used like voltameters; and dependence of E.M.F. on current . .......... 347

22. Summary of condensed statements embodying the writer's own

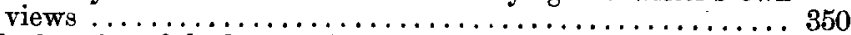

23. Explanation of the last of the above statements. Example of a Peltier series and of a Thomson series; and question whether thermoelectric contact-force depends upon chemical tendency or whether it is purely physical

24. Brief summary of the argument relating to the seat of E.M.F. in a pile .................................... 357

19. HAVING now explained why I believe the main part 11 of the Volta-effect to take its rise at the surface of

* Very little of this chapter, and none of Chapter V., has yet been read anywhere. 